\title{
Electro-osmotic properties of porous permeable films
}

\author{
Elena F. Silkina $\odot,{ }^{1}$ Naren Bag $\odot,^{2}$ and Olga I. Vinogradova $\oplus^{1,2, *}$ \\ ${ }^{1}$ Frumkin Institute of Physical Chemistry and Electrochemistry, Russian Academy of Sciences, \\ 31 Leninsky Prospect, 119071 Moscow, Russia \\ ${ }^{2}$ DWI-Leibniz Institute for Interactive Materials, Forckenbeckstr. 50, 52056 Aachen, Germany
}

(Received 13 March 2020; revised 26 June 2020; accepted 9 November 2020; published 1 December 2020)

\begin{abstract}
Permeable porous coatings on a flat solid support significantly impact its electrostatic and electrokinetic properties. Existing work has employed the mean-field PoissonBoltzmann theory by focusing on simplified cases, such as weakly charged and/or thick porous films compared to the extension of an electrostatic diffuse layer. In this paper, we obtain a closed-form analytical solution for electrostatic potential profiles by lifting the assumptions of both a small volume charge density and a thick film. Our analysis provides a framework for interpreting and predicting superproperties specific to porous films of an arbitrary thickness, from an enhanced ion absorption to a consequent amplification of electro-osmotic flows due to emergence of slip velocity at an interface with an outer electrolyte leading to a large zeta potential. The latter can be tuned by varying the amount of added salt and remains finite at even high concentrations. Our theory is valid for systems obeying the nonlinear Poisson-Boltzmann equation and the results are relevant for hydrogel coatings, porous carbon and silica, polyelectrolyte brushes, and more.
\end{abstract}

DOI: 10.1103/PhysRevFluids.5.123701

\section{INTRODUCTION}

Charged porous materials that are permeable to water and ions, such as polyelectrolyte networks, ion-exchange resins, silica gels, porous membranes, and electrodes, have found use in a large body of applications including water desalination [1], tissue engineering [2,3], and electrochemical systems [4]. Thanks to a recently discovered extremely strong electrokinetic flow near porous surfaces [5], new opportunities in microfluidics and advanced colloid technologies are emerging. Porous films on a variety of supports are similarly capable of providing such properties as improved transport and storage capacities for ions that they did not have when impermeable. However, the quantitative understanding of novel equilibrium and transport properties, which could not be achieved without porosity, is still challenging.

Considerable progress has been made over the last decades in understanding the equilibrium properties of porous surfaces in electrolyte solutions. Analytic solutions based on a linearized Poisson-Boltzmann theory are known [6-9], but these results do not apply to highly charged coatings, where nonlinear electrostatic effects could become significant. The nonlinear electrostatic problem has been treated using numeric and semianalytic approaches [7,10,11], and some simple analytic expressions for the static surface potential $\Psi_{s}$ have been derived for thick coatings compared to the Debye screening length, $\lambda_{D}$, which is a measure of the thickness of the electrostatic diffuse layer $[7,12]$. Nevertheless, approaches to calculate $\Psi_{s}$ analytically are sill lacking and general

\footnotetext{
*Corresponding author: oivinograd@yahoo.com
} 


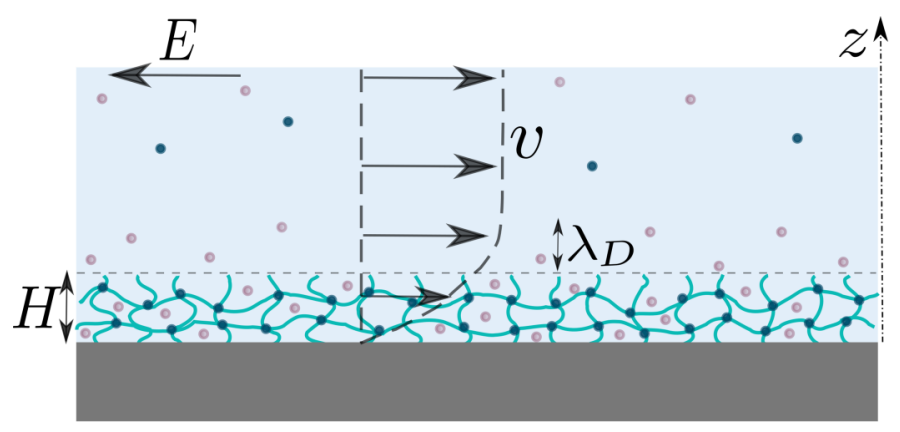

FIG. 1. Porous film of thickness $H$ in contact with an electrolyte solution. Anions and cations are denoted with bright and dark circles. An outer electrostatic diffuse layer of a thickness of the order of $\lambda_{D} \equiv \kappa^{-1}$ is formed in the vicinity of the coating. A tangential electric field, $E$, leads to a solvent flow of velocity $v$ (shown by right arrows).

principles to control it have not yet been established in the case of strongly charged coatings of a thickness smaller or comparable to $\lambda_{D}$.

The electrokinetic properties of porous surfaces in electrolyte solutions are relatively less understood, although there is some literature describing attempts to provide a theory of electro-osmosis near porous surfaces. It has been found that $\Psi_{s}$ of porous surfaces does not define unambiguously the electro-osmotic flow properties [6], and that bulk velocity is controlled, besides $\Psi_{s}$, by the Brinkman and inner Debye screening lengths [8]. These authors, however, failed to propose a physical interpretation of their results, which generally indicate that porous surfaces can amplify electro-osmotic pumping at micron scales, where pressure-driven flows are suppressed by viscosity. These theories and subsequent attempts at their improvement [10,13-15] are often invoked in the interpretation of the electro-osmotic data, but their relation to electrokinetic (zeta) potential, $Z$, which is the measure of electrokinetic mobility, has remained somewhat obscure. Some authors concluded that it "loses its significance" [8] or "is undefined and thus nonapplicable" [14], while others reported that $Z$ typically exceeds $\Psi_{s}[11,16]$. Recent analysis has included a more systematic treatment of the zeta potential of the porous coatings and proposed a mechanism of its enhancement, but is suitable for thick films only [17]. Thus, crucial aspects of the electrokinetics near highly charged porous films of an arbitrary thickness have been given insufficient attention so far, so they still remain poorly understood.

In this paper, we present a solution of the nonlinear Poisson-Boltzmann equation, which describes analytically the profiles of potentials induced by a planar porous coatings of any thickness, from very thin to thick compared to the Debye length. Our simple analytic expression is valid even when the volume charge density of a coating is quite large, and can be used for any salt concentration. From this theory, we are interpreting enhanced absorption properties of porous films, and show that due to these mobile absorbed ions the electro-osmotic flow inside the porous film emerges. The latter, in turn, leads to the finite slip velocity at the porous interface, which is the reason for an enhancement of the electro-osmotic velocity in the bulk electrolyte and of zeta potential. Finally, we obtain an upper bound on an attainable zeta potential that provides guidance for a giant amplification of electro-osmotic flows.

\section{ELECTRO-OSMOTIC EQUILIBRIA}

The system geometry is shown in Fig. 1. The permeable film of a thickness $H$ and a fixed volume charge density $\varrho$ (taken positive without loss of generality) is placed in a 1:1 electrolyte solution of bulk concentration $c_{\infty}$ and permittivity $\varepsilon$. To describe electrostatics of the system, we employ the classical nonlinear Poisson-Boltzmann approach [18], but the porous film is permeable for ions of 
the electrolyte solution, so that its potential builds up self-consistently. In this approach, ions obey Boltzmann distribution, $c_{ \pm}(z)=c_{\infty} \exp [\mp \psi(z)]$, where $\psi(z)=e \Psi(z) /\left(k_{B} T\right)$ is the dimensionless electrostatic potential, $e$ is the elementary positive charge, $k_{B}$ is the Boltzmann constant, $T$ is the temperature, and the upper (lower) sign corresponds to the cations (anions). The inverse Debye screening length of an electrolyte solution, $\kappa \equiv \lambda_{D}^{-1}$, is defined as $\kappa^{2}=8 \pi \ell_{B} c_{\infty}$, with the Bjerrum length $\ell_{B}=\frac{e^{2}}{\varepsilon k_{B} T}$. Note that as any approximation, the Poisson-Boltzmann formalism has its limits of validity but it always describes very accurately the ionic distributions for monovalent ions in the concentration range from $10^{-6}$ to $10^{-1} \mathrm{~mol} / \mathrm{L}[18]$.

\section{A. Electrostatic potentials}

The profile of a potential, $\psi(z)$, inside the porous film and in the outer solution satisfies the nonlinear Poisson-Boltzmann equation, which for our geometry can be formulated as

$$
\psi_{i, o}^{\prime \prime}=\kappa^{2}\left[\sinh \psi_{i, o}-\rho \Theta(H-z)\right],
$$

where ' denotes $d / d z$, with the index $\{i, o\}$ standing for in $(z \leqslant H)$ and out $(z \geqslant H), \rho=\frac{\varrho}{2 e c_{\infty}}$, and the Heaviside step function $\Theta(z)$. The solution of the Poisson-Boltzmann equation with prescribed boundary conditions, in general, requires a numerical method since it is nonlinear. Below we show that Eq. (1) can be integrated and propose its closed-form analytical solution. Although formally this solution is approximate, we will see that for all $\kappa H$ and $\rho$ it practically coincides with exact numerical calculations.

Integrating Eq. (1) twice by applying conditions $\psi_{o}^{\prime} \rightarrow 0$ and $\psi_{o} \rightarrow 0$ at $z \rightarrow \infty$, we find that the $\psi_{o}$-profile is identical to that near an impenetrable wall with the same surface potential [18],

$$
\psi_{o}=4 \operatorname{artanh}\left[\gamma e^{-\kappa(z-H)}\right]
$$

where $\gamma=\tanh \frac{\psi_{s}}{4}$ and $\psi_{s}=\psi(H)$ is the surface potential. Using $\psi_{i}^{\prime}(0)=0$ and $\psi_{i}(0)=\psi_{0}$, we obtain that $\psi_{0}$ and $\psi_{s}$ are related as

$$
\psi_{s} \equiv \psi_{0}-\frac{\cosh \psi_{0}-1}{\rho}
$$

derived before only for a limit of $\kappa H \gg 1$, i.e., for thick films [12]. Note that Eqs. (2) and (3) are exact and valid for any $\kappa H$ and $\rho$.

Further insight can be gained by recalling that local osmotic pressure of an electrolyte solution is $P=k_{B} T c$, where $c(z)=c_{+}(z)+c_{-}(z)$ is the total concentration of ions at given $z$. This clarifies that $\cosh \psi$ represents a dimensionless local osmotic pressure, $p=P / 2 c_{\infty} k_{B} T$, which takes its largest value of $p_{0}=\cosh \psi_{0}$ at $z=0$. Since $p(\infty)=1$, Eq. (3) indicates that an excess osmotic pressure at the wall, $p_{0}-1$, grows linearly with a self-induced potential difference across the porous film, $\psi_{0}-\psi_{s}$. It will be clear below that this is a main parameter that ascertains most of its properties.

In the limit of a thin film, $\kappa H \ll 1$, the asymptotic approach suggested before [19] can be employed. Expanding the potential in Eq. (1) about $z=0$, we obtain, to second order in $\kappa z$ :

$$
\psi_{i}(z) \simeq \psi_{0}-\frac{\rho}{2}(\kappa z)^{2}\left[1-\frac{\sinh \psi_{0}}{\rho}\right] .
$$

Note that the value of $\sinh \left(\psi_{0}\right) / \rho \leqslant 1$ represents the degree of screening of the film intrinsic charge at $z=0$.

One can find $\psi_{s}$ by substituting $z=H$ into Eq. (4). It follows then from Eq. (3) that $\psi_{0}$ satisfies

$$
\rho^{2}-\rho \sinh \psi_{0}-\frac{2\left(\cosh \psi_{0}-1\right)}{(\kappa H)^{2}} \simeq 0
$$


and standard manipulations then yield

$$
\psi_{0} \simeq \ln \left[\frac{2+(\rho \kappa H)^{2}+\rho \kappa H \sqrt{4+(\kappa H)^{2}\left(1+\rho^{2}\right)}}{2+\rho(\kappa H)^{2}}\right] .
$$

Consequently, the inner $\psi$-profile of a thin film is given by

$$
\psi_{i}(z) \simeq \psi_{s}-\frac{\left[\sinh \psi_{0}-\rho\right]}{2} \kappa^{2}\left(H^{2}-z^{2}\right)
$$

where $\psi_{s}$ and $\psi_{0}$ are described by Eqs. (3) and (6). Note that in some cases these two equations can be simplified. For instance, when $\rho \gg 1$, Eq. (6) may be reexpressed as

$$
\psi_{0} \simeq \ln \left[\frac{\left(\frac{\rho \kappa H}{2}+\sqrt{1+\left(\frac{\rho \kappa H}{2}\right)^{2}}\right)^{2}}{1+\frac{\rho(\kappa H)^{2}}{2}}\right],
$$

which is equivalent to

$$
\psi_{0} \simeq 2 \operatorname{arsinh}\left(\frac{\rho \kappa H}{2}\right)-\ln \left(1+\frac{\rho(\kappa H)^{2}}{2}\right) .
$$

Besides, $\psi_{s}$ and $\psi_{0}$ given by Eqs. (3) and (6) can be expanded in series for small $\rho \kappa H$, and we can easily find that in this case the $\psi$-profile is almost constant throughout the film:

$$
\psi_{0} \simeq \psi_{s} \simeq \rho \kappa H .
$$

The startling conclusion from analysis of Eq. (5) is that it is also valid for $\kappa H \gg 1$, i.e., for thick films. Indeed, in this case the last term on its left-hand side becomes very small compared with the first two, and we obtain a well known for thick films result [7]:

$$
\psi_{0}=\operatorname{arsinh}(\rho) .
$$

Note that the last equation immediately follows from Eq. (1) if we set $\psi_{i}^{\prime \prime}=0$, which is equivalent to a constant inner potential. This constant potential portion of thick films is globally electroneutral since absorbed electrolyte ions completely screen the intrinsic film charge. We recall that the potential of such an electroneutral area is usually referred to as the Donnan potential, $\psi_{D}$, so for thick films $\psi_{0} \equiv \psi_{D}$. As a side note, for thin films $\sinh \psi_{0} / \rho$ is normally smaller than 1 , as follows from Eq. (5), so $\psi_{0}$ should be below the Donnan potential of the porous medium. Returning to thick films, we note that Eq. (3) can be transformed to

$$
\psi_{s}=\psi_{0}+\frac{1-\sqrt{1+\rho^{2}}}{\rho} .
$$

Near the surface, electrolyte ions screen volume charges of the thick coating only partly, and an inner diffuse layer is formed. The inverse inner screening length can be found as

$$
\kappa_{i}=\kappa\left(\cosh \psi_{0}\right)^{1 / 2} \equiv \kappa \sqrt{p_{0}} .
$$

From Eq. (11), it then follows that $\kappa_{i} \simeq \kappa\left(1+\rho^{2}\right)^{1 / 4}$, which indicates that when $\rho \ll 1$, a sensible approximation should be $\kappa_{i} \simeq \kappa$. However, when $\rho \gg 1, \kappa_{i} \simeq \kappa \sqrt{\rho}$, and the criterion of a thick film can be relaxed to $\kappa H \sqrt{\rho} \gg 1$.

Since the thick film behaves as an electrolyte solution of the inverse Debye length $\kappa_{i}$, to obtain the exact equation for $\psi_{i}$ it is enough to simply change the variables in Eq. (2). Namely, substitution of $z$ by $-z, \psi_{s}$ by $\psi_{0}-\psi_{s}$, and $\psi_{o}$ by $\psi_{0}-\psi_{i}$ would immediately give

$$
\psi_{0}-\psi_{i}=4 \operatorname{artanh}\left[\gamma_{i} e^{-\kappa_{i}(H-z)}\right],
$$






FIG. 2. A distribution of a potential built up by a film of $\rho=10$, calculated numerically for $\kappa H=0.3$ (dashed curve) and 3 (solid curve). Filled circles correspond to calculations from Eqs. (7) and (14), when $z / H \leqslant 1$, and from Eq. (2), when $z / H \geqslant 1$. Open squares are obtained from Eq. (15).

where $\gamma_{i}=\tanh \frac{\psi_{0}-\psi_{s}}{4}$. Using Eqs. (11) and (12), we obtain $\gamma_{i}=\tanh \left(\frac{\sqrt{1+\rho^{2}}-1}{4 \rho}\right)$, which reduces to $\gamma_{i} \simeq \rho / 8$ if $\rho \ll 1$, and $\gamma_{i} \simeq \frac{1}{4}\left(1-\frac{1}{\rho}\right)$ when $\rho \gg 1$. This implies that $\gamma_{i}$ is always smaller than $1 / 4$. For such a small $\gamma_{i}$, the inner potential can be expanded about $\psi_{0}$, and to first order in $\psi_{0}-\psi_{s}$ we obtain

$$
\psi_{i}(z) \simeq \psi_{0}+\left(\psi_{s}-\psi_{0}\right) e^{\kappa_{i}(z-H)} .
$$

This derivation differs from conventional arguments, which assume low volume charge density [7]. Our treatment clarifies that Eq. (15) constitutes a sensible approximation for $\psi_{i}$ of a thick film of any $\rho$.

To assess the validity of the above approach, we employ numerical simulations. We perform a numerical resolution of a multipoint boundary value problem for the nonlinear Poisson-Boltzmann differential Eq. (1) with prescribed boundary conditions, using the numerical approach based on the collocation method [20].

In Fig. 2, we plot $\psi(z / H)$ computed for two different values of $\kappa H$ that are close to limits of thick and thin films, and a large fixed $\rho$. The form of the $\psi$-profile depends on $\kappa H$. For $\kappa H=3$, the inner potential shows a distinct plateau, indicating that the intrinsic charge of the film is completely screened by electrolyte ions, i.e., global electroneutrality. The plateau potential is equal to $\psi_{D}$, as discussed above. However, when $\kappa H=0.3$, there is no electroneutral region inside the film, and the potential at wall, $\psi_{0}$, is, as expected, much smaller than $\psi_{D}$. Also included in Fig. 2 are theoretical results obtained from Eqs. (2), (7), (14), and (15) and we conclude that in relevant areas they are in excellent agreement with numerical data.

It is tempting to speculate that Eq. (6) will be applicable for any $\kappa H$, and that a more elegant result, Eq. (9), can be used provided $\rho$ is large enough. Clearly, Eq. (5) could become less accurate for intermediate $\kappa H$, and it is of considerable interest to determine its regime of validity. To test ansatz (5), numerical and theoretical $\psi_{0}$ and $\psi_{s}$ have been calculated as a function of $\kappa H$ for $\rho=2$ and 20. Specimen results are plotted in Fig. 3, demonstrating the unprecedented accuracy of Eqs. (6) and (9) for all $\kappa H$ at chosen (large) values of $\rho$. As expected, Eq. (10), which can also be obtained using linear theory [7], is valid only when $\rho \kappa H$ is very small and significantly overestimates potentials, which saturate at some $\kappa H$, in other cases.

The charge density dependence of $\psi_{0}$ and $\psi_{s}$ is also of some interest. Figure 4 illustrates the growth of $\psi_{0}$ and $\psi_{s}$ with $\rho$ for the films of $\kappa H=0.2$ and 3. It is well seen that for a thinner film $\psi_{0} \simeq \psi_{s}$ up to $\rho \kappa H \simeq 1$. On increasing $\rho$ further, $\psi_{0}-\psi_{s}$ increases. For a thick film of $\kappa H=3$, the drop of a potential in the inner diffuse layer is always finite and $\psi_{0}-\psi_{s} \simeq 1$ as $\rho$ is increased. We again conclude that Eq. (6) fits accurately the numerical data. So does Eq. (9), except for $\rho \leqslant 1$, 


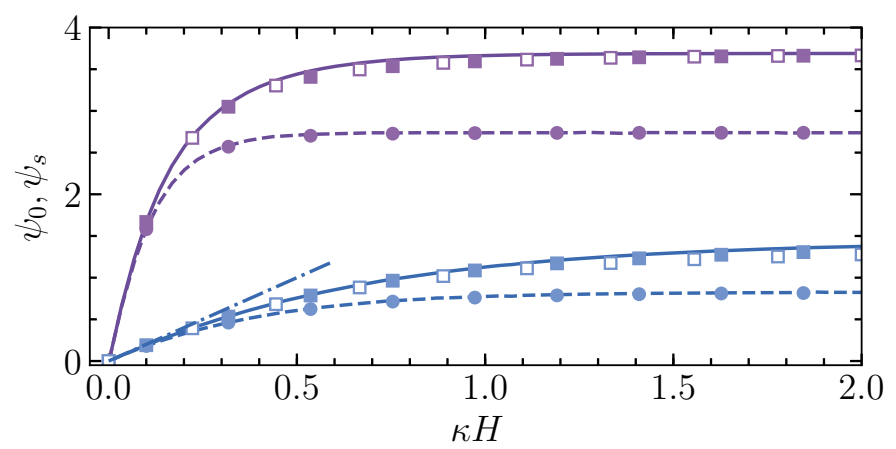

FIG. 3. Potentials at wall (solid lines) and surface (dashed) as a function of $\kappa H$ computed for fixed $\rho=20$ (upper set of curves) and $\rho=2$ (lower curves). Filled squares illustrate calculations from Eq. (6), circles are then obtained using Eq. (3). Open squares show results obtained using Eq. (9). Dash-dotted line is calculated from Eq. (10).

where some very small discrepancy is observed. Below we use Eq. (9) for all calculations [together with exact Eq. (3) for $\psi_{s}$ ] by omitting a discussion of its accuracy.

\section{B. Ion concentrations}

The problem we address here is the calculation of the profile of a cloud of counter-ions inside the porous film and in the electrolyte solution. Since ions obey Boltzmann distribution, their local concentrations $c_{ \pm} / c_{\infty}=\exp (\mp \psi)$ are determined solely by the $\psi$-profile calculated above.

Representative concentration profiles computed for films of $\rho=10$ and two different values of $\kappa H$ are shown in Fig. 5. Also included are theoretical calculations of $c_{ \pm} / c_{\infty}$ using $\psi_{o}$ obtained from Eq. (2) and $\psi_{i}$ given by Eqs. (7) and (14). We see that in the inner region, anions are significantly enriched and cations are depleted. The degree of this enrichment and depletion depends on the values of $\rho$ and $\kappa H$. At the given $\rho$, the degree of enrichment is ca. 20 for a thick film of $k H=5$ but is a few times smaller when $k H=0.2$. Note that in the latter case the concentration of anions is nonuniform throughout the film. We also stress that for a chosen value of $\rho$, inner concentrations of cations practically vanish for both $\kappa H$.

To boost absorption of ions, coatings of larger $\rho$ can be used, as illustrated in Fig. 6, where the total concentration of ions at the wall, $c_{0}=c_{+}(0)+c_{-}(0)$, scaled by the sum of anion and cation

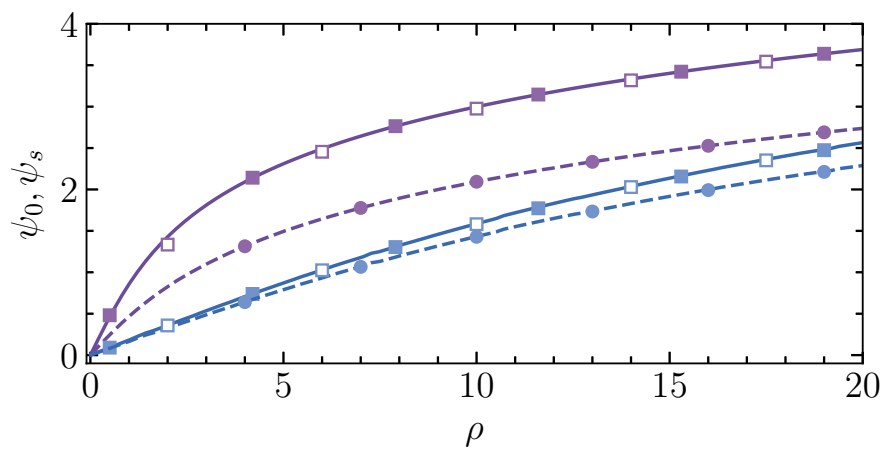

FIG. 4. Potentials at wall (solid lines) and surface (dashed) as a function of $\rho$ computed for fixed $\kappa H=3$ (upper set of curves) and $\kappa H=0.2$ (lower curves). Filled squares illustrate calculations from Eq. (6), circles are then obtained using Eq. (3). Open squares show predictions of Eq. (9). 


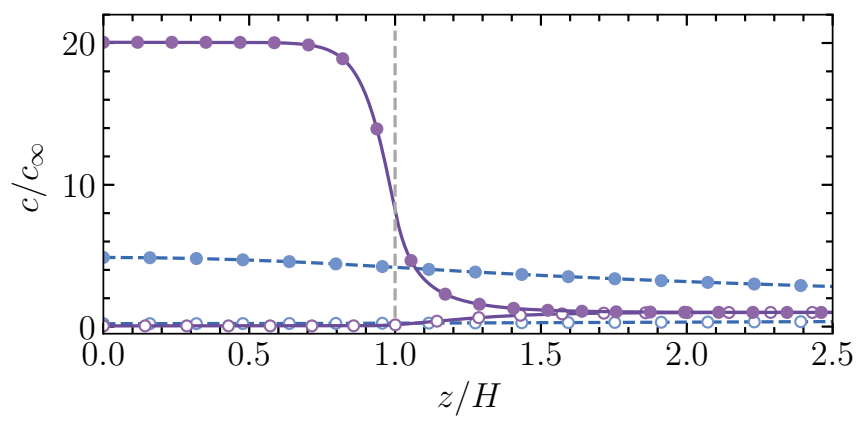

FIG. 5. Ion concentration profiles computed at $\rho=10$ using $\kappa H=0.2$ (dashed curves) and 5 (solid curves). Local concentrations of anions (filled circles) and cations (open circles) are calculated using Eq. (2) when $z / H \geqslant 1$, and from Eqs. (7) and (14) when $z / H \leqslant 1$.

concentrations at infinity, $2 c_{\infty}$, is plotted as a function of $\rho$. We note that $c_{0} / 2 c_{\infty}=\cosh \psi_{0} \equiv p_{0}$. It follows from Eq. (11) that for thick films $p_{0}=\sqrt{1+\rho^{2}}$. This equation describes perfectly numerical data for $\kappa H=3$, and it is clear that this curve corresponds to an upper attainable value of ion enrichment at the wall. In other words, the absorption capacity cannot be further improved by making the coating thicker. Note that $p_{0} \simeq 1+\rho^{2} / 2$ at small $\rho$, and $p_{0} \simeq \rho$ at large $\rho$. This is exactly what we observe for an upper curve in Fig. 6. However, the decrease in $\kappa H$ could significantly reduce the concentration of absorbed anions, especially when $\rho \leqslant 5$. The $c_{0} / 2 c_{\infty}$ curve computed for $\kappa H=0.3$ is included in Fig. 6 together with theoretical results obtained using Eq. (6). It can be seen, it is located much lower than the curve for a thick film. An expansion of $\psi_{0}$ defined by Eq. (6) for small $\kappa H$ leads to $p_{0} \simeq 1+(\rho \kappa H)^{2} / 2$ that fits the lower curve in Fig. 6 very well when $\rho \leqslant 5$ (not shown). Clearly, an excess osmotic pressure, $p_{0}-1$, is always extremely small for thin films of $\rho=O(1)$ since it scales with $(\rho \kappa H)^{2}$, indicating their low absorption capacity.

\section{ELECTROOSMOTIC VELOCITY AND ZETA POTENTIAL}

Another relevant problem is an electro-osmotic flow of a solvent of the dynamic viscosity $\eta$ in an applied tangential electric field, $E$. The origin of the electro-osmotic flow is traditionally attributed to diffuse layers [21]. For porous coatings, the electroosmosis is defined both by diffuse layers and absorbed ions and, as we will see, the second mechanism is responsible for a flow amplification and dominates at high salt.



FIG. 6. Ion-enrichment at the wall, $c_{0} / 2 c_{\infty}=\cosh \psi_{0}$, vs $\rho$ computed using $\kappa H=3$ (upper curve) and 0.3 (lower curve). Open and filled circles indicate results obtained using $\psi_{0}$ calculated from Eqs. (6) and (11). 
We are now about to relate the dimensionless velocity, $v(z)=\frac{4 \pi \ell_{B} \eta}{e E} V(z)$, of such a flow to $\psi_{0}$ and $\psi_{s}$. We assume a weak field, so in the steady state, $\psi(z)$ is independent of the fluid flow. Since for our planar geometry the concentration gradients at every location are perpendicular to the direction of the flow, it is legitimate to neglect advection. Therefore, the liquid flow satisfies the generalized Stokes equation

$$
v^{\prime \prime}-\mathcal{K}^{2} v \Theta(H-z)=\psi^{\prime \prime}+\kappa^{2} \rho \Theta(H-z),
$$

where $\mathcal{K}$ is the inverse Brinkman length. At the wall, we apply a classical no-slip condition, $v_{0}=$ $v(0)=0$, and far from the surface $v_{z \rightarrow \infty}^{\prime}=0$. We consider the limits of small flow extension into the porous medium, $\mathcal{K} H \rightarrow \infty$, and of $\mathcal{K} H \rightarrow 0$, where an additional dissipation in the porous film can be neglected, to obtain bounds on the electro-osmotic velocity that constrain its attainable value.

From analysis of Eq. (16), it follows that the outer $v$-profile and velocity in the bulk are given by

$$
v_{o}(z)=v_{\infty}+\psi_{o}(z), \quad v_{\infty}=v_{s}-\psi_{s}=-\zeta,
$$

where $\psi_{o}$ is defined by Eq. (2), $v_{s}=v(H)$ is the liquid velocity at surface-below we refer it to as slip velocity-and $\zeta=e Z /\left(k_{B} T\right)$. Equations (17) indicate that enhanced electro-osmotic mobility can be a consequence of large equilibrium $\psi_{s}$, as well as of large (negative for a positively charged coating we consider here) $v_{s}$ that depends on the hydrodynamic permeability of the coating. The amplification of the electro-osmotic flow (compared to the no-slip case with the same $\psi_{s}$ ) can be expressed as

$$
\frac{\zeta}{\psi_{s}}=1-\frac{v_{s}}{\psi_{s}}
$$

The problem thus reduces to calculation of $v_{s}$. Below we provide analytical results together with exact numerical calculations.

If we suppose $\mathcal{K} H \rightarrow \infty$, the slip velocity nearly vanishes, $\psi_{s} \simeq \zeta$, and $v_{\infty} \simeq-\psi_{s}$, which is equivalent to the Smoluchowski result. When $\mathcal{K} H \rightarrow 0$, integrating Eq. (16) twice, and imposing the continuity of $v$ and $v^{\prime}$ at $z=H$, we find

$$
v_{i} \simeq\left(\psi_{i}-\psi_{0}\right)-\rho \kappa^{2}\left(H z-\frac{z^{2}}{2}\right),
$$

where for films of any thickness $\psi_{0}$ is given by Eq. (6). The first term reflects the reduction of the potential inside the porous coating. The second term is associated with a body force $\rho \kappa^{2}$ that drives the inner flow by acting on the accumulated mobile ions. This contribution resembles the usual no-slip parabolic Poiseuille flow. It follows from Eq. (19) that $-v_{s} \simeq \psi_{0}-\psi_{s}+\frac{\rho(\kappa H)^{2}}{2}$. Since $\psi_{0}-\psi_{s} \leqslant 1$, the second term should dominate even at moderate $\rho$.

The outer velocity $v_{o}$ is given by Eqs. (17) with

$$
v_{\infty} \simeq-\left(\psi_{0}+\frac{\rho(\kappa H)^{2}}{2}\right) \simeq-\zeta .
$$

Figure 7(a) includes typical numerical and theoretical $v$-profiles calculated for this case using $\kappa H=1$ and $\rho=5$ that leads to $\psi_{s} \simeq 1.47$. Since with these parameters $\kappa_{i} \simeq 2.26$, such a film is of intermediate thickness, i.e., it is neither thin nor thick. When $\mathcal{K} H \rightarrow \infty$, the flow is the same as near the no-slip impenetrable wall, and the sole role of a porous film is to set $\psi_{s}$. The $v$-profile for $\mathcal{K} H \rightarrow 0$ is also shown. It turns out that even at moderate $\rho$ and $\kappa H$ taken here, one can induce significantly enhanced $v_{\infty}$, which is associated with the emergence of a large slip velocity, $v_{s}$. Also included in Fig. 7(a) is the curve computed using $\mathcal{K} H=2$. With such a value of $\mathcal{K} H$, the Brinkman length of a porous material of a given film (of $\kappa H=1)$ is two times smaller than $\lambda_{D}$. We see that the computed curve is located between two limiting cases and demonstrates quite large $v_{s}$.

We now verify Eq. (20) and plot theoretical $v_{\infty}$ vs $\kappa H$ in Fig. 7(b) together with numerical data. Upon increasing $\kappa H$ at fixed $\rho$, the amplitude of $v_{\infty}$ grows nonlinearly, and in the case of $\rho \gg 1$ 

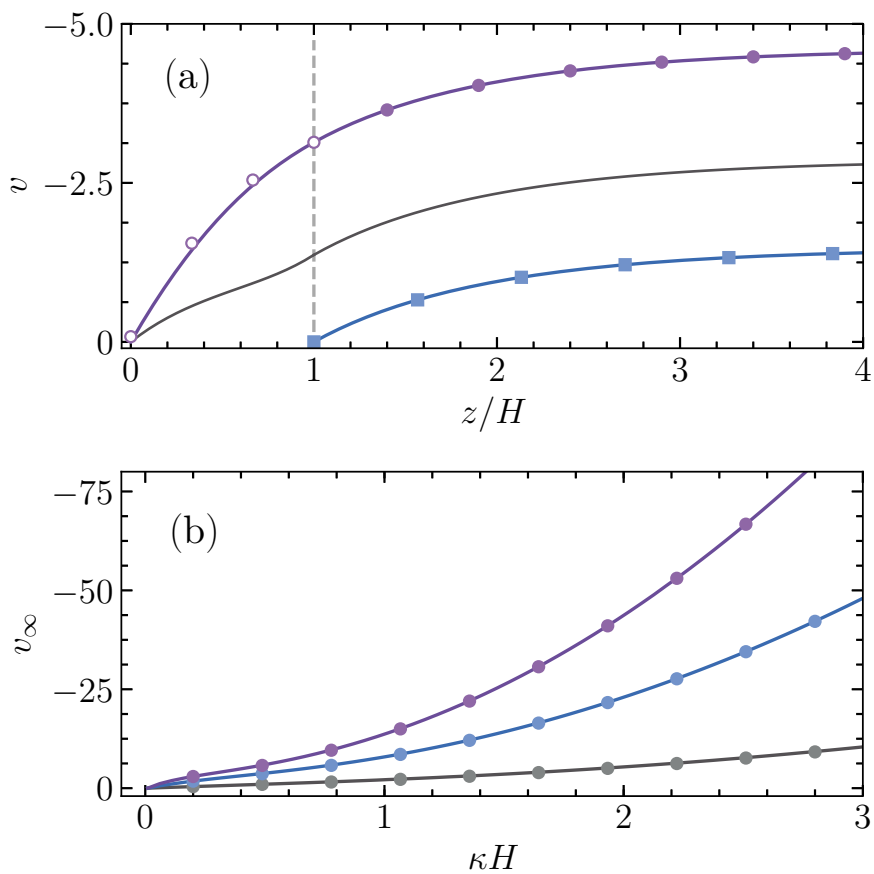

FIG. 7. (a) Electroosmotic velocity profiles computed using $\kappa H=1, \rho=5$, and $\mathcal{K} H=0,2$, and $\infty$ (solid curves from top to bottom). Filled and open symbols indicate calculations from Eqs. (17) and (19). Squares are obtained using $v_{s}=0$, circles correspond to $v_{s}=\psi_{s}+v_{\infty}$, where $v_{\infty}$ is given by Eq. (20); (b) computed upper bounds $(\mathcal{K} H \rightarrow 0)$ on $v_{\infty}$ vs $\kappa H$ (solid curves). From top to bottom $\rho=20,10$, and 2. Circles show results of calculations from Eq. (20).

becomes several tens of times faster compared to a no-slip case, even at moderate $\kappa H$. It is tempting to speculate that one can further amplify $v_{\infty}$, making porous film thicker. However, when the film becomes thick enough, the condition $\mathcal{K} H \rightarrow 0$ violates, and Eq. (20) is no longer valid. We note that the magnitude of $v_{\infty}$ also grows on increasing $\rho$, confirming predictions of Eq. (20). Finally, we mention that calculations made from Eq. (20) with $\psi_{0}$ calculated from Eq. (6) perfectly fit the numerical data.

These results point strongly that mobile ions absorbed within the porous layer actively participate in the flow-driving mechanism by reacting to the field. The porous film acts as a charged immobile surface layer with absorbed mobile ions of the opposite sign, but note the difference from a known example of mobile surface charges at the slippery wall [22]. In the latter case, slippage is of a hydrodynamic origin and mobile surface charges induce a backward flow, reducing the amplification of electro-osmotic flow caused by hydrodynamic slip. By contrast, in the current work an inner solvent flow induces a forward flow and $v_{s}$ itself. However, similarly to hydrophobic electrokinetics [22,23], our large $\zeta$ no longer reflects the sole $\psi_{s}$. Finally, we would like to stress that a massive amplification of electro-osmotic flow that can be achieved near porous surfaces is of the same order of that at charged superhydrophobic surfaces [24], and such a fast flow is in agreement with recent observations [5].

So far, we have considered the potentials and electro-osmotic velocity at fixed dimensionless $\rho$ and $\kappa H$. Additional insight into the problem can be gleaned by calculating an upper bound on $\zeta$ as a function of $c_{\infty} \propto \kappa^{2}$ at fixed $H$ and $\varrho$. Let us now keep fixed $H=25 \mathrm{~nm}$ and $\varrho=150 \mathrm{kC} / \mathrm{m}^{3}$, usually referred to as moderate [25], and vary $c_{\infty}$ from $10^{-5}$ to $10^{-2} \mathrm{~mol} / \mathrm{L}$. Upon increasing $c_{\infty}$ in this range, $\kappa H$ is increased from about 0.25 to 8 , and $\rho$ is reduced from about 78 down to 0.1 . 




FIG. 8. $\zeta$ as a function of $c_{\infty}$ computed for a film of $H=25 \mathrm{~nm}, \varrho=150 \mathrm{kC} / \mathrm{m}^{3}$, using $\mathcal{K} H=0,1.5$, and $\infty$ (solid curves from top to bottom). Dashed curve shows $\left|v_{s}\right|$ at $\mathcal{K} H=0$. Squares, open and filled circles plot results of calculations from Eqs. (21)-(23).

Therefore, for a given film, the required regimes (e.g., of thin and thick films, or highly and weakly charged coatings) can be tuned simply by adjusting the concentration of salt.

The bounds on $\zeta$ are shown by lower and upper curves in Fig. 8. If $\mathcal{K} H \rightarrow \infty, \zeta \simeq \psi_{s}$ decays from ca. $5\left(Z \simeq 125 \mathrm{mV}\right.$ ) practically to zero as $c_{\infty}$ increases, leading to a suppression of a flow. In dilute solutions, the lower bound is given by

$$
\zeta \simeq \ln \left(\frac{\varrho}{e c_{\infty}}\right)-1
$$

which perfectly fits numerical data. When $\mathcal{K} H \rightarrow 0, \zeta$ becomes much larger. Thus, with our smallest concentration, $\zeta \simeq 8$ (or $Z \simeq 200 \mathrm{mV}$ ). In a dilute solution,

$$
\zeta \simeq \ln \left(\frac{\varrho}{e c_{\infty}}\right)+\frac{2 \pi \ell_{B} \varrho H^{2}}{e}
$$

where the first term is associated with the potential at the wall. Note that such a logarithmic decay fits well the obtained for real porous materials data [5]. For concentrated solutions, a large, and independent on salt, zeta potential is observed. The computed slip velocity, also included in Fig. 8, indicates that this occurs when $\zeta \simeq-v_{s}$ and $\psi_{s} \simeq 0$. Thus, a large zeta potential emerges solely due to a forward electro-osmotic flow inside a porous film. It is easy to show that it is given by

$$
\zeta \simeq \frac{2 \pi \ell_{B} \varrho H^{2}}{e}
$$

which clarifies the status of second term in Eq. (22). This result is relevant for the understanding zeta-potential measurements with "hairy" surfaces, where it remains finite even at high salt concentrations [6,26]. We recall that Eqs. (21) and (22) represent the lower and upper bounds for $\zeta$ attained at limiting values of $\mathcal{K} H$. Any finite $\mathcal{K} H$ would lead to $\zeta$ confined between the above bounds, as seen in Fig. 8, where we use $\mathcal{K} H=1.5$.

\section{CONCLUSION}

Using the Poisson-Boltzmann approach, we derived simple analytic equations that provide considerable insight into the electro-osmotic equilibria and flows in the presence of porous coatings of an arbitrary thickness, and are suitable even when they are strongly charged. Our theory describes absorption capacity of porous films, which is, in turn, responsible for an enhanced electro-osmotic flow. The bounds on zeta potential we have derived can guide the design of coatings to amplify and control electrokinetic phenomena. We have also shown that electrokinetic properties of the porous films can easily be tuned by adjusting the concentration of an electrolyte solution. 
Since the Poisson-Boltzmann formalism is a mean-field approximation, corrections to our theory, such as, for instance, due to correlations and fluctuations of charge densities, may play a substantial role for multivalent electrolyte ions or for concentrations above $10^{-1} \mathrm{~mol} / \mathrm{L}$. It would be of some interest to extend our work to account for these and other possible deviations from the mean-field description.

\section{ACKNOWLEDGMENTS}

We thank E.S.Asmolov for helpful discussions. This work was supported by the Ministry of Science and Higher Education of the Russian Federation and by the German Research Foundation (Grant No. Vi 243/4-2) within the SPP 1726 Microswimmers-From Single Particle Motion to Collective Behaviour.

[1] S. Porada, L. Weinstein, R. Dash, A. van der Wal, M. Bryjak, Y. Gogotsi, and P. M. Biesheuvel, Water desalination using capacitive deionization with microporous carbon electrodes, ACS Appl. Mater. Interfaces 4, 1194 (2012).

[2] D. Stamatialis, B.J. Papenburg, M. Gironas, S. Saiful, S.N.M. Bettahalli, S. Schmitmeier, and M. Wessling, Medical applications of membranes: Drug delivery, artificial organs and tissue engineering, J. Membrane Sci. 308, 1 (2008).

[3] H. H. Chung, M. Mireles, B. J. Kwarta, and T. R. Gaborski, Use of porous membranes in tissue barrier and co-culture models, Lab Chip 18, 1671 (2018).

[4] P. M. Biesheuvel, Y. Fu, and M. Z. Bazant, Diffuse charge and Faradaic reactions in porous electrodes, Phys. Rev. E 83, 061507 (2011).

[5] D. Feldmann, P. Arya, T. Y. Molotilin, N. Lomadze, A. Kopyshev, O. I. Vinogradova, and S. A. Santer, Extremely long-range light-driven repulsion of porous microparticles, Langmuir 36, 6994 (2020).

[6] E. Donath and V. Pastushenko, Eletrophoretical study of cell surface properties. The influence of the surface coat on the electric potential distribution and on general electrokinetic properties of animal cells, Bioelectrochem. Bioenerg. 6, 543 (1979).

[7] H. Ohshima and S. Ohki, Donnan potential and surface potential of a charged membrane, Biophys. J. 47, 673 (1985).

[8] H. Ohshima and T. Kondo, Electrokinetic flow between two parallel plates with surface charge layers: Electro-osmosis and streaming potential, J. Colloid Interface Sci. 135, 443 (1990).

[9] S. Chanda, S. Sinha, and S. Das, Streaming potential and electroviscous effects in soft nanochannels: Towards designing more efficient nanofluidic electrochemomechanical energy converters, Soft Matter 10, 7558 (2014).

[10] J. F. L. Duval, Electrokinetics of diffuse soft interfaces. 2. Analysis based on the nonlinear PoissonBoltzmann equation, Langmuir 21, 3247 (2005).

[11] G. Chen and S. Das, Streaming potential and electroviscous effects in soft nanochannels beyond DebyeHückel linearization, J. Colloid Interface Sci. 445, 357 (2015).

[12] E. F. Silkina, T. Y. Molotilin, S. R. Maduar, and O. I. Vinogradova, Ionic equilibria and swelling of soft permeable particles in electrolyte solutions, Soft Matter 16, 929 (2020).

[13] H. Ohshima, Electrophoresis of soft particles, Adv. Colloid Interface Sci. 62, 189 (1995).

[14] J. F. L. Duval and H. P. van Leeuwen, Electrokinetics of diffuse soft interfaces. 1. Limit of low Donnan potentials, Langmuir 20, 10324 (2004).

[15] H. Ohshima, Theory of Colloid and Interfacial Electric Phenomena (Elsevier, Amsterdam, 2006).

[16] V. D. Sobolev, A. N. Filippov, T. A. Vorob'eva, and I. P. Sergeeva, Determination of the surface potential for hollow-fiber membranes by the streaming-potential method, Colloid J. 79, 677 (2017).

[17] O. I. Vinogradova, E. F. Silkina, N. Bag, and E. S. Asmolov, Achieving large zeta-potentials with charged porous surfaces, Phys. Fluids 32, 102105 (2020). 
[18] D. Andelman, Soft Condensed Matter Physics in Molecular and Cell Biology (Taylor \& Francis, New York, 2006), Chap. 6.

[19] E. F. Silkina, E. S. Asmolov, and O. I. Vinogradova, Electro-osmotic flow in hydrophobic nanochannels, Phys. Chem. Chem. Phys. 21, 23036 (2019).

[20] G. Bader and U. Ascher, A new basis implementation for a mixed order boundary value ODE solver, SIAM J. Sci. Stat. Comput. 8, 483 (1987).

[21] J. L. Anderson, Colloid transport by interfacial forces, Annu. Rev. Fluid Mech. 21, 61 (1989).

[22] S. R. Maduar, A. V. Belyaev, V. Lobaskin, and O. I. Vinogradova, Electrohydrodynamics Near Hydrophobic Surfaces, Phys. Rev. Lett. 114, 118301 (2015).

[23] L. Joly, C. Ybert, E. Trizac, and L. Bocquet, Hydrodynamics within the Electric Double Layer on Slipping Surfaces, Phys. Rev. Lett. 93, 257805 (2004).

[24] A. V. Belyaev and O. I. Vinogradova, Electro-Osmosis on Anisotropic Super-Hydrophobic Surfaces, Phys. Rev. Lett. 107, 098301 (2011).

[25] J. F. L. Duval, R Zimmermann, A L Cordeiro, N Rein, and C Werner, Electrokinetics of diffuse soft interfaces. IV. Analysis of streaming current measurements at thermoresponsive thin films, Langmuir 25, 10691 (2009).

[26] A. Garg, C. A. Cartier, K. J. M. Bishop, and D. Velegol, Particle zeta potentials remain finite in saturated salt solutions, Langmuir 32, 11837 (2016). 